\title{
Cierre cognitivo, sexismo y religiosidad: diferencias y similitudes entre grupos de adolescentes con distinta cultura
}

\author{
Manuel Moyano*1, Francisca Expósito ${ }^{2}$ y Humberto M. Trujillo \\ ${ }^{1}$ Universidad de Córdoba \\ ${ }^{2}$ Universidad de Granada
}

\begin{abstract}
Resumen: Desde el ámbito de la cognición social motivada, la necesidad de cierre cognitivo emerge como una variable crucial para la mejor comprensión de las diferencias individuales en variables psicosociales asociadas a la cultura y, además, susceptible de tener en cuenta en programas de in tervención cuyo objetivo vaya dirigido a modificar actitudes ancladas en el prejuicio y la discriminación. En la presente investigación se ha realizado un estudio de este constructo motivacional y su relación con el sexismo y la religiosidad en una muestra de adolescentes cristianos y musulmanes, todos ellos residentes en un contexto social con un marcado carácter multicultural. De acuerdo con los datos obtenidos, no se han encontrado diferencias entre musulmanes y cristianos en necesidad de cierre cognitivo y sexismo. Sin embargo, se han encontrado diferencias por sexo en sexismo hostil. La necesidad de cierre cognitivo correlacionó de una forma estadísticamente significativa con el sexismo (hostil y benevolente), y es un buen predictor. Los musulmanes puntuaron de una forma significativamente más elevada que los cristianos en los indicadores de religiosidad. Se discuten los resultados en el marco de investigaciones previas.

Palabras clave: Cierre cognitivo; sexismo; religiosidad; cultura.
\end{abstract}

Title: Cognitive closure, sexism and religiosity: differences and similarities between teenagers with different culture.

Abstract: In the context of motivated social cognition, the need for cognitive closure emerges like a decisive factor for a better understanding of individual differences on psychosocial variables linked to culture. Besides, they are likely to be taken into account in intervention programmes which objectives would aim to modify attitudes anchored in prejudice and discrimination. The goal of this research is to study this motivational construct and its relationship with sexism and religiosity with a sample of Christians and Muslims teenagers, all of them living in a multicultural context. According to the gathered data, there are not any significant differences between Muslims and Christians in need for cognitive closure and sexism. However, there are significant differences between girls and boys in hostile sexism. The need for cognitive closure was positively related, in a statistically significant way, with sexism (hostile and benevolent), and it is a significant predictor. Muslims got higher scores than Christians on indicators of religiosity in a statistically significant way. Results are discussed in relation to previous research.

Key words: Cognitive closure; sexism; religiosity; culture.

\section{Introducción}

El concepto de necesidad de cierre cognitivo (need for cognitive closu$r e)$, fue propuesto por Arie W. Kruglanski (1989, 2004) para desarrollar un modelo científico sobre los aspectos cognitivo-conductuales de la toma de decisiones. Básicamente, la necesidad de cierre cognitivo puede ser definida como el deseo de dar una respuesta rápida a una pregunta o cuestión que tiene contenido confuso y ambiguo. Se asume que la magnitud de la necesidad de cierre cognitivo está determinada por los beneficios percibidos del hecho de cerrar y por los costes asociados a no cerrar. Esta idea se basa en dos aspectos relacionados: por un lado, la tendencia a la "urgencia", esto es, a concluir los estados de carencia de cierre cognitivo con premura; y por otro, la tendencia a la "permanencia", esto es, a tratar de mantener dichos estados de cierre cognitivo bloqueando las hipótesis alternativas iniciales.

Se ha constatado que la necesidad de cierre cognitivo puede ser incrementada temporalmente por manipulaciones del contexto (ruido, presión temporal, hacinamiento). Así, la necesidad de cierre cognitivo será más elevada en circunstancias donde conservar dicho cierre sea demasiado costoso o supusiera un laborioso procesamiento de información, tal y como puede ocurrir en condiciones de presión temporal, en ambientes ruidosos o cuando la persona esté fatigada, intoxicada o bajo el efecto de drogas. Pero, además, las perso-

* Dirección para correspondencia [Correspondence address]:

Manuel Moyano. Departamento de Psicología. Universidad de Córdoba.

C/ San Alberto Magno s/n. 14041 Córdoba (España).

E-mail: mmoyano@uco.es nas también difieren sustancialmente en sus niveles de tendencia personal al cierre cognitivo, lo que sugiere que varía a lo largo de un continuo. En suma, en la necesidad de cierre cognitivo existen diferencias individuales que son susceptibles de ser evaluadas y medidas. Concretamente, Webster y Kruglanski $(1994,1998)$ han elaborado y aplicado una escala para evaluar este constructo que ha sido traducida a numerosas lenguas y ha mostrado validez discriminante con manipulaciones situacionales y otros constructos de interés. Posteriormente, Roets y Van Hiel (2007) realizaron algunas modificaciones a la escala original y, más recientemente, en 2011, validaron una versión breve de 15 ítems de dicho instrumento. En general, las aportaciones empíricas respecto a las diferencias individuales en este constructo apuntan a que los individuos con una elevada necesidad de cierre cognitivo prefieren el orden y la estructura en sus vidas (evitando en lo posible el desorden y el caos), la previsibilidad (optando por conocimiento seguro y estable), experimentan un deseo urgente en la toma de decisiones, muestran incomodidad por la ambigüedad (experimentándola como aversiva) y tienen tendencia a una mayor cerrazón mental.

A día de hoy se han aportado una gran cantidad de investigaciones empíricas en relación con este concepto en diferentes niveles de análisis: intrapersonal, interpersonal, intragrupal e intergrupal. También sabemos que diferentes ideologías de carácter conservador se han relacionado con una elevada necesidad de cierre cognitivo. A modo de ejemplo, esta variable se ha relacionado directamente con el autoritarismo de derechas, la orientación a la dominancia social, cognitiva, e inversamente con la necesidad de cognición o la el racismo sutil y el manifiesto o la necesidad de estructura 
necesidad de afecto. Además, existen evidencias empíricas de que el cierre cognitivo guarda relación con formas de prejuicio, tales como el sexismo, y con ciertas variables religiosas, especialmente con indicadores de fundamentalismo religioso (puede consultarse una revisión reciente en Kruglanski, Dechesne, Orehek y Pierro, 2009).

En relación a las asociaciones entre el cierre cognitivo y el sexismo, diferentes estudios empíricos han constatado que la necesidad de cierre es un predictor importante del sexismo. Así, Doherty (1998) examinó cómo las motivaciones para el cierre cognitivo pueden influir en las reacciones de los miembros de un grupo a la conformidad y la desviación ancladas en expectativas de roles de género. En el contexto de la teoría de la justificación del sistema, Calogero y Jost (2011) realizaron varios experimentos en los que encontraron que, ante la exposición a ciertos estímulos sexistas, evitar la necesidad de cierre podía desempeñar en las mujeres un importante papel protector de cara a asumir para sí mismas ciertas ideologías sexistas. Otro marco teórico de interés para encontrar evidencias empíricas de la potencial relación entre el constructo de necesidad de cierre cognitivo y el sexismo podría ser la teoría del sexismo ambivalente (Glick y Fiske, 1996, 2001). Desde dicha perspectiva se distingue entre sexismo hostil y sexismo benévolo, distinguiéndose cada uno de estos tipos de sexismo por diferentes aspectos. Por un lado, el sexismo hostil se refiere al sexismo tradicional, basado en la supuesta inferioridad de las mujeres como grupo. Por otro, el sexismo benévolo asume una visión de la mujer como ser débil y necesitado de protección con unos roles estereotipados claramente restringidos, pero desde una perspectiva más positiva, siendo el tono emocional más afectivo y suscitando en los hombres conductas de protección e incluso de adoración hacia las mujeres. En ese marco, cabe decir que Pek y Leong (2003) encontraron una correlación positiva entre la necesidad de cierre y el sexismo hostil.

Con respecto a las relaciones entre el cierre cognitivo y la religión, se ha postulado que la necesidad de cierre podría estar relacionada con la religiosidad, especialmente con indicadores de fundamentalismo religioso, aunque esta relación está lejos de ser sencilla (Hill, Cohen, Terrell y Nagoshi, 2010). No obstante, hay que decir que el fundamentalismo religioso se ha relacionado con una gran variedad de constructos asociados a la necesidad de manejar la incertidumbre y de disponer de estructuras epistémicas sólidas. Así, se ha encontrado que los extremistas religiosos son más dogmáticos y más autoritarios (Altemeyer, 2002; Altemeyer y Hunsberger, 1992, 2004), con mayores niveles de necesidad de cierre (Saroglou, 2002) y más tendentes a mostrar prejuicio hacia una variedad de exogrupos (incluido el prejuicio anclado en el sexo), los cuales pueden llegar a ser discriminados en un esfuerzo por proteger la certidumbre cognitiva que proporciona una ideología extrema (Brandt y Reyna, 2010).

Además de lo anteriormente expuesto, en diferentes trabajos se han examinado la relación entre religiosidad y sexismo. Y más específicamente, se ha sugerido la importancia de la variable género en la comprensión de la relación en- tre la religión y el sexismo (Maltby, Hall, Anderson y Edwards, 2009). En algunos trabajos de corte asociativo, como el realizado por Glick, Lameiras y Rodríguez (2002), se ha encontrado que, tanto para hombres como para mujeres, la religiosidad católica se relaciona más con el sexismo benévolo que con actitudes sexistas de carácter hostil. De igual forma, Burn y Busso (2005) también encontraron que diferentes indicadores de religiosidad estaban asociados con el sexismo benévolo, pero no con el hostil. Estos datos son consistentes con la idea de que la participación activa de ciertas religiones puede reforzar ideologías sexistas que legitiman la desigualdad de género.

Por último, si bien existen un gran número de teorías e investigaciones empíricas que han abordado los factores contribuyentes a la exclusión social y los procesos de aculturación inmigrante, en el estudio de tales dinámicas no ha sido habitual analizar conjuntamente el sexismo, las diferencias culturales asociadas a la religión y la necesidad de cierre cognitivo (Berry, 2001; Hitti, Mulvey y Pillen, 2011; Kosic, Kruglanski, Pierro y Mannetti, 2004; Navas, Rojas y García, 2011). En base a lo expuesto, en la presente investigación se pretende estudiar dicho constructo motivacional y su relación con el sexismo y la religiosidad, así como las interrelaciones entre éstos; factores psicosociales, todos ellos, muy dependientes de la cultura y de permanente actualidad. Para ello, se ha realizado un trabajo de campo de carácter exploratorio en un contexto multicultural con una elevada validez ecológica y donde conviven adolescentes musulmanes y cristianos en proporciones similares.

Los objetivos generales de la investigación son los siguientes: (1) estudiar las diferencias y similitudes que existen entre distintos grupos sociales (musulmanes y cristianos, chicos y chicas) en la necesidad de cierre cognitivo, sexismo y diferentes variables religiosas; (2) analizar las relaciones funcionales entre dichas variables.

\section{Método}

\section{Participantes}

Los participantes en el estudio residen en un barrio marginal de una capital española caracterizado por una considerable diversidad multicultural y en el que se estima que viven unas 9.000 personas. La muestra se ha obtenido del único instituto de educación secundaria existente en dicho barrio, estando conformada por 115 alumnos y alumnas. La edad estaba comprendida entre los 13 y los 17 años, siendo la media aritmética de 14.7 años. Con respecto al sexo, 62 son chicos y 53 son chicas. De los 115 adolescentes, 66 se autodefinen como musulmanes y 49 como cristianos. Con respecto a los musulmanes, decir que 60 se autodefinen como extranjeros y seis como españoles hijos de extranjeros. En relación a la práctica religiosa, hay que decir que el 100\% de los musulmanes se autodefinen como practicantes de su religión, mientras que se autodefinen como practicantes el $61 \%$ de los cristianos. 


\section{Instrumentos}

Need for Closure Scale (Webster y Kruglanski, 1994; adaptado al español por Moyano, 2011; ver también una adaptación breve al español en Horcajo, Díaz, Gandarillas y Briñol, 2011) es una escala conformada por 42 ítems que evalúa la necesidad de cierre, entendida como la motivación para alcanzar respuestas que estén alejadas de la confusión y la ambigüedad. Los ítems deben ser puntuados en una escala comprendida entre 1 (totalmente en desacuerdo) y 6 (totalmente de acuerdo). En la presente investigación se utilizará la puntuación media total de los 42 ítems del instrumento. La consistencia interna, obtenida mediante el alfa de Cronbach, fue de .92 .

Escala de Detección de Sexismo en Adolescentes (Recio, Cuadrado y Ramos, 2007). Esta escala, compuesta por 26 ítems, sirve para detectar el sexismo en adolescentes y permite diferenciar entre sexismo hostil (16 ítems) y benévolo (10 ítems). Los ítems deben ser puntuados en una escala comprendida entre 1 (totalmente en desacuerdo) y 6 (totalmente de acuerdo). La consistencia interna de las subescalas de sexismo hostil y benévolo en el presente estudio fue de un alfa de Cronbach de .92 y de .78, respectivamente, siendo la fiabilidad del total de la escala de .93 .

Preguntas sobre posicionamiento religioso de elaboración propia para la presente investigación. Concretamente, se pidió a los participantes que señalaran en una escala tipo Likert de 0 a 6 el grado en que "la religión era importante en la vida", el grado en que pensaban que "la religión propia tenía valores morales mejores que los de las otras religiones" y el "miedo a la muerte".

Periodicidad de asistencia a la mezquita o a la iglesia. El formato de respuesta de esta última pregunta adoptó una escala tipo Likert de 1 a 7 , donde 1 equivale a "nunca" y 7 a "todos los días, varias veces al día".

Por último, se registró información de variables sociodemográficas tales como la edad, el sexo, el nivel de estudios, la religión, si eran o no practicantes de la misma y la nacionalidad.

\section{Procedimiento}

La aplicación de los instrumentos de evaluación se realizó en marzo de 2010. La participación en el estudio fue totalmente voluntaria. Previamente, se había solicitado el permiso correspondiente al equipo directivo del centro educativo. El encargado de aplicar el instrumento fue un investigador entrenado para ello. La aplicación duró aproximadamente 40 minutos y se realizó en las aulas habituales del alumnado, contando con la colaboración del profesorado del centro.

\section{Análisis de datos}

En primer lugar, se calcularon los estadísticos descriptivos y las correlaciones de las variables psicosociales evaluadas. En segundo lugar, se analizó la frecuencia y el porcentaje según sexo y religión en la periodicidad de asistencia a la mezquita o iglesia. En tercer lugar, se realizó un Análisis Multivariado de la Varianza (MANOVA), tomando como factores fijos el sexo y la religión. Se empleó el valor de la traza de Pillai para observar si existían diferencias estadísticamente significativas en todas las variables dependientes analizadas conjuntamente. Se utilizó el estadístico Eta cuadro $\left(\eta^{2}\right)$ parcial como estimación del tamaño del efecto. En cuarto lugar, se realizaron varios modelos de regresión lineal con el fin de evaluar el poder explicativo de algunas variables del estudio. Los datos fueron analizados con el paquete estadístico SPSS 17.0.

\section{Resultados}

\section{Estadísticos descriptivos y relaciones entre las varia- bles}

En primer lugar, en la Tabla 1 se presentan los estadísticos descriptivos de tendencia central, variabilidad, forma de las variables evaluadas en el estudio y la correlación entre las mismas.

Tabla 1. Estadísticos descriptivos y correlaciones entre las variables $(N=115)$.

\begin{tabular}{|c|c|c|c|c|c|c|c|c|c|c|}
\hline & $M$ & $D T$ & Asimetria & Curtosis & 1 & 2 & 3 & 4 & 5 & 6 \\
\hline 1. Necesidad cierre cognitivo & 3.64 & .68 & -.25 & .08 & & & & & & \\
\hline 2. Sexismo hostil & 3.35 & 1.19 & -.09 & -.49 & $.511 * *$ & & & & & \\
\hline 3. Sexismo benévolo & 3.81 & .91 & -.23 & .49 & $.727^{* *}$ & $.719 * *$ & & & & \\
\hline 4. Importancia religión & 4.84 & 1.47 & -1.21 & .50 & -.019 & -.097 & -.058 & & & \\
\hline 5. Religión propia valores mejores & 4.17 & 1.88 & -.47 & -1.24 & .093 & $.386^{* *}$ & $.221 *$ & $.246 * *$ & & \\
\hline 6. Miedo muerte & 3.27 & 2.05 & .08 & -1.67 & -.113 & -.032 & .090 & -.140 & -.117 & \\
\hline 7. Periodicidad mezquita / iglesia & 2.90 & 2.27 & .592 & -1.36 & .179 & $.331 * *$ & .052 & $.404 * *$ & $.420 * *$ & $-.270 * *$ \\
\hline
\end{tabular}

Nota: ${ }^{*} p<.05 ;{ }^{* *} p<.01$

Los estadísticos descriptivos obtenidos son adecuados técnicamente para el uso investigador. Las puntuaciones de asimetría y curtosis se encontraron dentro del rango de normalidad. Con respecto a las correlaciones entre las variables psicosociales evaluadas, hay que decir que la necesidad de cierre mostró correlaciones elevadas y estadísticamente significativas con el sexismo hostil y el benévolo. Las subescalas de sexismo hostil y benévolo mostraron también una asociación moderada $(r=.719 ; p<.01)$. El sexismo hostil mostró correlaciones estadísticamente significativas con la creencia de que la religión propia tiene valores mejores $(r=$ $.386 ; p<.01)$ y con la periodicidad de asistencia a la mezqui- 
ta o iglesia $(r=.331 ; p<.01)$. Además, cabe destacar que la periodicidad de asistencia a la mezquita o iglesia mostró correlaciones positivas y estadísticamente significativas con la importancia dada la religión $(r=.404 ; p<.01)$ y con la creencia de que la religión propia tiene valores mejores $(r=$ $.420 ; p<.01)$. También correlacionó de una forma negativa con el miedo a la muerte $(r=-.270 ; p<.01)$.
Diferencias en la asistencia a la mezquita y a la iglesia

En la Tabla 2 se muestra la frecuencia y el porcentaje de la periodicidad de asistencia a la mezquita y a la iglesia distinguiendo por sexos.

Tabla 2. Periodicidad de asistencia a mezquita o iglesia según el sexo y la religión.

\begin{tabular}{|c|c|c|c|c|}
\hline \multirow[b]{2}{*}{ Periodicidad mezquita } & \multicolumn{2}{|c|}{ Chicos musulmanes $(N=31)$} & \multicolumn{2}{|c|}{ Chicas musulmanas $(N=35)$} \\
\hline & Frecuencia & $\%$ & Frecuencia & $\%$ \\
\hline Nunca & 1 & 3.2 & 26 & 74.3 \\
\hline Una vez al mes & 0 & 0 & 2 & 5.7 \\
\hline Una vez cada quince días & 0 & 0 & 1 & 2.9 \\
\hline Una vez a la semana & 9 & 29.0 & 1 & 2.9 \\
\hline Tres o cuatro días a la semana & 2 & 6.5 & 2 & 5.7 \\
\hline Todos los días & 12 & 38.7 & 3 & 8.6 \\
\hline \multirow[t]{2}{*}{ Todos los días, varias veces al día } & 7 & 22.6 & 0 & 0 \\
\hline & \multicolumn{2}{|c|}{ Chicos cristianos $(N=31)$} & \multicolumn{2}{|c|}{ Chicas cristianas $(N=18)$} \\
\hline Periodicidad iglesia & Frecuencia & $\%$ & Frecuencia & $\%$ \\
\hline Nunca & 24 & 77.4 & 10 & 55.6 \\
\hline Una vez al mes & 2 & 6.5 & 2 & 11.1 \\
\hline Una vez cada quince días & 1 & 3.2 & 0 & 0 \\
\hline Una vez a la semana & 0 & 0 & 0 & 0 \\
\hline Tres o cuatro días a la semana & 2 & 6.5 & 4 & 22.2 \\
\hline Todos los días & 1 & 3.2 & 2 & 11.1 \\
\hline Todos los días, varias veces al día & 1 & 3.2 & 0 & 0 \\
\hline
\end{tabular}

En el grupo de musulmanes, prácticamente todos los chicos, excepto uno, acuden a la mezquita al menos una vez a la semana. Además, en torno al $60 \%$ de los chicos acude diariamente a la mezquita. Por el contrario, el $74 \%$ de las chicas musulmanas no acuden nunca a la mezquita. Solamente 6 de ellas asisten con alguna periodicidad a la mezquita. En el grupo de cristianos, la asistencia a la iglesia está mucho menos mediatizada por la variable sexo, existiendo por tanto, diferencias sustanciales con respecto a los musulmanes. En general, en el grupo de cristianos la asistencia periódica a la iglesia es un comportamiento infrecuente. De hecho, el 77\% de los chicos y el 55\% de las chicas no asisten nunca, y son una minoría los que acuden a diario.

\section{Diferencias en función del sexo y la religión}

Previamente, con el fin de contrastar la hipótesis nula de que las matrices de covarianza observadas de las variables dependientes son iguales en todos los grupos, se realizó la prueba de Box $(M$ de Box $=191.376 ; F=1.51 ; p<.002)$. Asimismo, con el fin de contrastar el supuesto de homogeneidad de varianzas se realizó el contraste de Levene, el cual sólo resultó significativo con el ítem que evaluaba que "la re- ligión propia tenía valores morales mejores que los de las otras religiones" $(F=4.64 ; p<.006)$.

El MANOVA reveló diferencias estadísticamente significativas en función del sexo (Traza de Pillai $=.462 ; F=4.9 ; p$ $<$.000). En la Tabla 3 se presentan las puntuaciones medias para las variables psicosociales evaluadas en función del sexo, así como las estimaciones del tamaño del efecto.

Como se puede apreciar, se encontraron diferencias estadísticamente significativas en sexismo hostil y en la periodicidad de asistencia a la mezquita o iglesia, variables donde los chicos obtuvieron una mayor puntuación media en comparación con las chicas.

El MANOVA también reveló diferencias estadísticamente significativas en función de la religión (Traza de Pillai = $.495 ; F=5.59 ; p<.000)$. En la Tabla 4 se presentan las puntuaciones medias para las variables en función de la religión, así como las estimaciones del tamaño del efecto.

Los musulmanes obtuvieron una mayor puntuación media en comparación con los cristianos en la importancia dada la religión en sus vidas, en la creencia de que su religión tenía valores morales mejores, así como en la periodicidad de asistencia a la mezquita o iglesia. Los cristianos puntuaron de una forma más elevada que los musulmanes en el miedo a la muerte. 
$\underline{\text { Tabla 3. Diferencias en función del sexo en las variables evaluadas }}$

\begin{tabular}{|c|c|c|c|c|c|c|c|}
\hline & \multicolumn{2}{|c|}{ Chicos } & \multicolumn{2}{|c|}{ Chicas } & \multirow[b]{2}{*}{$F$} & \multirow[b]{2}{*}{$p$} & \multirow[b]{2}{*}{$\eta^{2}$ parcial } \\
\hline & $M$ & $D T$ & $M$ & DT & & & \\
\hline Necesidad cierre cognitivo & 3.9 & .63 & 3.52 & .64 & 2.98 & .091 & .061 \\
\hline Sexismo hostil & 3.89 & 1.11 & 2.77 & 1.24 & 13.29 & .001 & .224 \\
\hline Sexismo benévolo & 3.99 & .91 & 3.64 & .99 & 1.28 & .263 & .027 \\
\hline Importancia religión & 4.29 & 1.48 & 5 & 1.23 & .69 & .408 & .015 \\
\hline Religión propia valores mejores & 4.04 & 1.79 & 3.68 & 1.98 & 1.87 & .178 & .039 \\
\hline Miedo a la muerte & 3.43 & 2.09 & 3.73 & 2.05 & 1.27 & .264 & .027 \\
\hline Periodicidad mezquita / iglesia & 3.58 & 2.39 & 2.09 & 1.83 & 13.6 & .001 & .228 \\
\hline
\end{tabular}

Tabla 4. Diferencias en función de la religión en las variables evaluadas.

\begin{tabular}{|c|c|c|c|c|c|c|c|}
\hline & \multicolumn{2}{|c|}{ Musulmanes } & \multicolumn{2}{|c|}{ Cristianos } & \multirow[b]{2}{*}{$F$} & \multirow[b]{2}{*}{$p$} & \multirow[b]{2}{*}{$\eta^{2}$ parcial } \\
\hline & $M$ & DT & $M$ & $D T$ & & & \\
\hline Necesidad cierre cognitivo & 3.59 & .62 & 3.86 & .67 & .93 & .338 & .020 \\
\hline Sexismo hostil & 3.48 & 1.38 & 3.32 & 1.21 & 1.89 & .176 & .039 \\
\hline Sexismo benévolo & 3.75 & .98 & 3.92 & .95 & .09 & .765 & .002 \\
\hline Importancia religión & 5.42 & 1.1 & 3.85 & 1.25 & 17.7 & .000 & .278 \\
\hline Religión propia valores mejores & 4.46 & 2 & 3.35 & 1.59 & 5.37 & .025 & .105 \\
\hline Miedo a la muerte & 2.96 & 2.05 & 4.12 & 1.94 & 5.10 & .029 & .100 \\
\hline Periodicidad mezquita / iglesia & 3.53 & 2.36 & 2.04 & 1.83 & 10.95 & .002 & .192 \\
\hline
\end{tabular}

Por último, el MANOVA aportó una interacción estadísticamente significativa entre el sexo y la religión (Traza de Pillai $=.313 ; F=2.6 ; p<.026)$, concretamente en la creencia de que la religión propia tenía valores morales mejores $(F$ $=6.4 ; p<.015 ; \eta^{2}$ parcial $\left.=.122\right)$ y en la periodicidad de asistencia a la mezquita o a la iglesia $\left(F=13.81 ; p<.001 ; \eta^{2}\right.$ parcial $=.231)$.

\section{Modelos predictivos}

Con el fin de examinar el poder explicativo de diferentes variables, se realizaron varios modelos predictivos. En la
Tabla 5 se presentan dos modelos de regresión jerárquica utilizando como criterios el sexismo hostil y el sexismo benévolo. En dichos análisis se introdujeron secuencialmente como predictores el sexo, la religión, la periodicidad de asistencia a la mezquita o iglesia y la necesidad de cierre cognitivo. Como se puede apreciar, en un primer paso, la variable sexo entró a formar parte de la ecuación de regresión como predictor exclusivamente en el sexismo hostil. Esto es algo que consideramos coherente teniendo en cuenta que, tal y como se ha ido aportando por numerosas investigaciones

Tabla 5. Análisis de regresión jerárquica sobre el sexismo hostil y benévolo.

\begin{tabular}{|c|c|c|c|c|c|c|c|c|}
\hline & \multicolumn{4}{|c|}{ Sexismo hostil } & \multicolumn{4}{|c|}{ Sexismo benévolo } \\
\hline & $\mathrm{R}^{2}$ & $\beta$ & $t$ & $p$ & $\mathrm{R}^{2}$ & $\beta$ & $t$ & $p$ \\
\hline Paso 1 & .211 & & & & .032 & & & \\
\hline Sexo & & -.459 & -3.83 & .000 & & -.18 & -1.37 & .173 \\
\hline Paso 2 & .241 & & & & .036 & & & \\
\hline Sexo & & -.520 & -4.14 & .000 & & -1.68 & -1.25 & .213 \\
\hline Religión & & .184 & 1.46 & .148 & & -.062 & -.46 & .647 \\
\hline Paso 3 & .331 & & & & .045 & & & \\
\hline Sexo & & -.375 & -2.87 & .006 & & -.111 & -.722 & .473 \\
\hline Religión & & .023 & .17 & .862 & & -.124 & -.784 & .437 \\
\hline Periodicidad mezquita / iglesia & & .348 & 2.66 & .010 & & .124 & .741 & .462 \\
\hline Paso 4 & .443 & & & & .531 & & & \\
\hline Sexo & & -.320 & -2.62 & .011 & & -.013 & -.119 & .906 \\
\hline Religión & & .103 & .824 & .414 & & .065 & .568 & .573 \\
\hline Periodicidad mezquita / iglesia & & .237 & 1.89 & .064 & & -.045 & -.372 & .711 \\
\hline Necesidad de cierre cognitivo & & .366 & 3.22 & .002 & & .743 & 7.483 & .000 \\
\hline Total $\mathrm{R}^{2} c$ & .4 & & & & .49 & & & \\
\hline
\end{tabular}

anteriores, incluyendo los resultados del MANOVA de esta investigación, las diferencias en sexismo benévolo entre hombres y mujeres son mucho menos evidentes (o incluso inexistentes) que las que se suelen encontrar en el sexismo hostil. En el segundo bloque de la ecuación de regresión se introdujo la religión, que no entró de forma estadísticamente 
significativa en el modelo. En un tercer bloque, se introdujo la periodicidad de asistencia a la mezquita o iglesia, entrando a formar parte de la ecuación de regresión de forma estadísticamente significativa como predictor del sexismo hostil. Por último, se introdujo la necesidad de cierre cognitivo. En este último modelo, el sexismo hostil es predicho de una forma estadísticamente significativa tanto por el sexo como por la necesidad de cierre cognitivo, manteniéndose de una forma marginalmente significativa la periodicidad de asistencia a la mezquita o iglesia. Con respecto al sexismo benévolo, la única variable predictora estadísticamente significativa fue la necesidad de cierre cognitivo. Este último modelo de regresión explicó un $40 \%$ de la varianza total en el caso del sexismo hostil y un 49\% en el caso del sexismo benévolo.

Posteriormente se realizaron otros análisis de regresión múltiple complementarios tomando como criterio el ítem que evaluaba la creencia de que "la propia religión tiene valores mejores que las otras religiones". En el grupo de cristianos, tanto el sexismo hostil $\left(\beta=.51 ; t=3.55 ; \mathrm{R}^{2} \mathrm{c}=.24 ; p\right.$ $<.001)$ como el benévolo $\left(\beta=.57 ; t=4.2 ; R^{2} \mathrm{c}=.31 ; p=\right.$ .000) resultaron buenos predictores de dicho ítem. En el grupo de musulmanes, únicamente el sexismo hostil parece tener alguna relación con la creencia de que la propia religión tiene valores mejores, $\left(\beta=.35 ; t=2.51 ; R^{2} \mathrm{c}=.1 ; p<\right.$ $.016)$.

\section{Discusión y conclusiones}

Consideramos que con este trabajo se han aportado diferentes hallazgos de interés y se han conseguido los objetivos inicialmente propuestos. En la muestra multicultural analizada no se encontraron diferencias estadísticamente significativas en la necesidad de cierre cognitivo en función del sexo o la religión. Tampoco hallamos diferencias significativas en sexismo (ni hostil ni benévolo) según la religión. Sin embargo, sí se han encontrado diferencias por sexo en sexismo hostil. Tal y como se ha puesto de manifiesto en investigaciones previas (Expósito, Moya y Glick, 1998; Lameiras y Rodríguez, 2002; Recio, Cuadrado y Ramos, 2007), no se obtuvieron diferencias estadísticamente significativas en sexismo benévolo en chicos y chicas.

Si bien la necesidad de cierre no mostró un patrón de correlaciones positivas con los indicadores de religiosidad evaluados, tal y como podría esperarse, éste constructo sí correlacionó de una forma estadísticamente significativa con el sexismo hostil y benévolo, y de hecho, resultó ser un buen predictor de dichas variables, independientemente del sexo y de la religión. Este dato nos hace pensar que el cierre cognitivo puede ser una variable muy relacionada con la ideología de género. En general, estos resultados son coherentes con otras investigaciones que también han encontrado una asociación positiva entre necesidad de cierre cognitivo y sexismo (Calogero y Jost, 2011; Doherty, 1998; Pek y Leong, 2003). Esto supone que las personas con alta necesidad de cierre cognitivo van a estar más predispuestas a apoyar este- reotipos de género que sirven para el mantenimiento de las desigualdades.

En relación a las diferencias según la religión, hay que destacar que mientras que todos los musulmanes se autodefinen como practicantes, sólo el $60 \%$ de los cristianos se considera practicante de su religión. Los musulmanes también puntuaron de una forma más elevada que los cristianos en los indicadores de religiosidad (importancia dada la religión y creencia de que la religión propia tenía valores morales mejores) y mostraron una mayor homogeneidad en las respuestas aportadas si tenemos en cuenta las desviaciones típicas. Por otra parte, la asistencia periódica a la mezquita o la iglesia está muy descompensada en musulmanes y cristianos respectivamente. Además, en el caso de los musulmanes esta conducta es poco habitual entre las chicas, al menos en el contexto social estudiado. En otras palabras, la asistencia a la mezquita en los musulmanes es un comportamiento muy dependiente del sexo, al menos en la muestra y en el contexto analizado. Consideramos que, aunque limitados, los datos en este sentido son muy relevantes debido a la escasez de información previa relativa a dicho comportamiento en entornos marginales. Como podemos imaginar, estas pautas van a tener implicaciones directas en las actitudes y comportamientos diferenciales en chicos y chicas en función de su religión. Todos los datos anteriores, analizados conjuntamente, son evidencias de que, aunque la tendencia social en los jóvenes españoles tiende a la secularización, existen nuevas formas de religiosidad vinculadas al fenómeno migratorio y a sus movimientos sociales en los que no parece darse esta tendencia, sino todo lo contrario.

Otro aspecto a destacar es la correlación negativa encontrada entre el miedo a la muerte y los indicadores de religiosidad, especialmente la periodicidad de asistencia a la mezquita o iglesia. Esto es coherente con un gran número de investigaciones que han evidenciado el papel de la religiosidad como protector de la ansiedad relacionada con la propia muerte (saliencia de mortalidad) y que la postre podría favorecer respuestas diferentes a las amenazas existenciales (Friedman y Rholes, 2007; Greenberg, Porteus, Simon, Pyszczynski y Solomon, 1995; Norenzayan, Dar-Nimrod, Hansen, y Proulx, 2009; Norenzayan y Shariff, 2008). Sin embargo, como ya hemos comentado no encontramos relaciones estadísticamente significativas entre necesidad de cierre cognitivo y la religiosidad en la muestra analizada.

Como se comentó en la introducción, en investigaciones previas se había descubierto una relación entre sexismo (especialmente la dimensión benévola del sexismo) y religiosidad, aunque esta relación está lejos de ser simple (Burn y Busso, 2005; Glick, Lameiras y Rodríguez, 2002). En esta investigación hemos obtenido resultados que muestran algunas particularidades destacables. Concretamente, nuestros modelos predictivos mostraron que, en el grupo de cristianos, tanto el sexismo hostil como el benévolo tenían relación positiva con la creencia de que "la propia religión tiene valores mejores que las otras religiones". Sin embargo, en el grupo de musulmanes se alteró la relación esperada, en- 
contrándose que exclusivamente el sexismo hostil (y no el benévolo) estaba relacionado con la religiosidad. Estos datos son una muestra más de que las actitudes y comportamientos religiosos y la ideología de género son aspectos psicosociales complejos; modulados, además de por el sexo y la religión, por el entorno social de referencia. Obviamente, dado que el estudio es correlacional, debemos ser precavidos y no establecer relaciones causales apresuradas entre estas variables.

Posiblemente, las limitaciones de esta investigación están asociadas al restringido tamaño de la muestra y a sus particularidades. Además, las características del contexto, un entorno urbano donde las relaciones intergrupales y la convivencia son extremadamente sensibles y potencialmente conflictivas, pueden haber supuesto ciertas peculiaridades en los resultados obtenidos. Por consiguiente, los mismos deben ser interpretados con cautela, dado que la muestra es singular y pueden estar influyendo otras variables sociodemográficas que, teniendo en cuenta la diversidad multicultural del entorno, difícilmente podían ser operacionalizadas sin generar suspicacias, reactividad y/o deseabilidad social en los participantes. Nos referimos a aspectos tales como la tendencia o movimiento religioso específico que se profesaba dentro de la adhesión a una religión concreta, el origen geográfico de las familias de los extranjeros musulmanes o la variable étnica "ser payo o gitano". En relación a este último aspecto, hay que decir que, en el contexto del barrio, residen un con-

\section{Referencias}

Altemeyer, B. (2002). Dogmatic behavior among students: Testing a new measure of dogmatism. Journal of Social Psychology, 142, 713-721.

Altemeyer, B. y Hunsberger, B. (1992). Authoritarianism, religious fundamentalism, quest, and prejudice. International Journal for the Psychology of Religion, 2, 113-133.

Altemeyer, B. y Hunsberger, B. (2004). A revised religious fundamentalism scale: The short and sweet of it. International Journal for the Psychology of Religion, 14, 47-54

Berry, J. W. (2001). A Psychology of Immigration. Journal of Social Issues, 57, 615-631.

Brandt, M. J. y Reyna, C. (2010). The Role of Prejudice and the Need for Closure in Religious Fundamentalism. Personality and Social Psychology Bulletin, 36, 715-725.

Burn, S. M. y Busso, J. (2005). Ambivalent Sexism, Scriptural Literalism, and Religiosity. Psychology of Women Quarterly, 29, 412-418.

Calogero, R. M. y Jost, J. J. (2011). Self-subjugation among women: Exposure to sexist ideology, self-objectification, and the protective function of the need to avoid closure. Journal of Personality and Social Psychology, 100, 211-228.

Doherty, K. T. (1998). A Mind of Her Own: Effects of Need for Closure and Gender on Reactions to Nonconformity. Sex Roles, 38, 801-819.

Expósito, F., Moya, M. y Glick, P. (1998). Sexismo ambivalente: medición y correlatos. Revista de Psicología Social, 13, 159-169.

Friedman, M. y Rholes, W. S. (2007). Successfully challenging fundamentalist beliefs results in increased death awareness. Journal of Experimental Social Psychology, 43, 794-801.

Glick, P. y Fiske, S. T. (1996). The ambivalent sexism inventory: Differentiating hostile and benevolent sexism. Journal of Personality and Social Psychology, 70, 491-512.

Glick, P. y Fiske, S. T. (2001). Ambivalent sexism. En M. P. Zanna (Ed.), Advances in experimental social psychology (pp. 115-188). San Diego: Academic Press. siderable número de personas de etnia gitana, así como una nada desdeñable cantidad de mestizos payos-gitanos. Estos matices étnicos e identitarios pueden estar influyendo, sin duda, en algunos de los resultados obtenidos, y deberían ser controlados en futuras investigaciones que se realicen en contextos más convencionales.

Con el fin de superar las limitaciones que ha podido suponer el hecho de utilizar medidas de un sólo ítem, en futuros trabajos sería conveniente utilizar escalas para evaluar la religiosidad, especialmente, los indicadores de religiosidad extrema. Es posible que, de esta forma, las relaciones de estas variables con la necesidad de cierre cognitivo también sean más concluyentes.

En España no han sido muchos los trabajos que han indagado en las relaciones entre el constructo de necesidad de cierre cognitivo con otras variables de interés para la psicología aplicada, tales como la religiosidad o el sexismo. Si conocemos mejor tales relaciones podremos tener más datos empíricos para desarrollar programas de intervención basados en la evidencia que puedan ser útiles para incidir en el cambio de actitudes de problemas sociales de interés. Todo apunta a que para reducir el prejuicio, modificar actitudes sexistas o inhibir la polarización intergrupal de personas y grupos sociales en contextos multiculturales deberíamos tener en cuenta las diferencias individuales existentes en dicho constructo motivacional.

Glick, P., Lameiras, M. y Rodríguez, Y. (2002). Education and Catholic Religiosity as Predictors of Hostil and Benevolent Sexism Toward Women and Men. Sex Roles, 47, 433-441.

Greenberg, J., Porteus, J., Simon, L., Pyszczynski, T. y Solomon, S. (1995). Evidence of a terror management function of cultural icons: The effects of mortality salience on the inappropriate use of cherished cultural symbols. Personality and Social Psychology Bulletin, 21, 1221-1228.

Hill, E. D., Cohen, A. B., Terrell, H. K. y Nagoshi, C.T. (2010). The Role of Social Cognition in the Religious Fundamentalism-Prejudice Relationship. Journal for the Scientific Study of Religion, 49, 724-739.

Hitti, A., Mulvey, K. L. y Killen, M. (2011). Social exclusion and culture: The role of group norms, group identity and fairness. Anales de Psicología, 27, 587-599.

Horcajo, J., Díaz, D., Gandarillas, B. y Briñol, P. (2011). Adaptación al español del Test de Necesidad de Cierre Cognitivo. Psicothema, 23, 864-870.

Kosic, A., Kruglanski, A. W., Pierro, A. y Mannetti, L. (2004). Social cognition of immigrants acculturation: Effects of the need for closure and the reference group at entry. Journal of Personality and Social Psychology, 86, 796-813.

Kruglanski, A. W. (1989). Lay epistemics and buman knowledge: Cognitive and motivational bases. New York: Plenum.

Kruglanski, A.W. (2004). The psychology of closed mindedness. New York: Psychology Press.

Kruglanski, A. W., Dechesne, M., Orehek, E. y Pierro, A. (2009). Three decades of lay epistemics: The why, how and who of knowledge formation. European Review of Social Psychology, 20, 146-191.

Lameiras, M. y Rodríguez, Y. (2002). Evaluación del sexismo moderno en adolescentes. Revista de Psicología Social, 17, 119-127.

Maltby, L. E., Hall, M. E. L., Anderson, T. L. y Edwards, K. (2009). Religion and Sexism: The Moderating Role of Participant Gender. Sex Roles, 62, 615-622. 
Moyano, M. (2011). Factores psicosociales contribuyentes a la radicalización islamista de jóvenes en España. Construcción de un instrumento de evaluación. (Tesis doctoral). Universidad de Granada.

Navas, M., Rojas, A. J. y García, M. C. (2011). Concordancia entre actitudes y percepciones de aculturación de la población autóctona hacia los inmigrantes magrebíes: relación con las actitudes prejuiciosas. Anales de Psicología, 27, 186-194.

Norenzayan, A., Dar-Nimrod, I., Hansen, I. G. y Proulx, T. (2009). Mortality salience and religion: Divergent effects on the defense of cultural worldviews for the religious and the non-religious. European Journal of Social Psychology, 39, 101-113.

Norenzayan, A. y Shariff, A. (2008). The Origin and Evolution of Religious Prosociality. Science, 322, 58-62.

Pek, J. C. X. y Leong, F. T. L. (2003). Sex-related self-concepts, cognitive styles and cultural values of traditionality-modernity as predictors of general and domain-specific sexism. Asian Journal of Social Psychology, 6, $31-49$.
Recio, P., Cuadrado, I. y Ramos, E. (2007). Propiedades psicométricas de la Escala de Detección de Sexismo en Adolescentes (DSA). Psicothema, 19, 522-528.

Roets, A. y Van Hiel, A. (2007). Separating ability from need: Clarifying the dimensional structure of the Need for Closure Scale. Personality and Social Psychology Bulletin, 33, 266-280.

Roets, A. y Van Hiel, A. (2011). Item selection and validation of a brief, 15item version of the Need for Closure Scale. Personality and Individual Differences, 50, 90-94.

Saroglou, V. (2002). Beyond dogmatism: the need for closure as related to religion. Mental Health, Religion and Culture, 5, 183-194.

Webster, D. M. y Kruglanski, A. W. (1994). Individual differences in need for cognitive closure. Journal of Personality and Social Psychology, 67, 10491062.

Webster, D. M. y Kruglanski, A. W. (1998). Cognitive and social consequences of the need for cognitive closure. European Review of Social Psychology, 8, 133-173.

(Artículo recibido: 9-9-2011, revisado: 27-03-2012, aceptado: 22-05-2012) 